\title{
Equilíbrio, controle postural e força muscular em idosas osteoporóticas com e sem quedas
}

\author{
Balance, postural control and muscle strength in \\ osteoporotic elderly women with and without falls \\ Sarah Rubia Ferreira de Meneses', Thomaz Nogueira Burke², Amélia Pasqual Marques ${ }^{3}$
}

\begin{abstract}
RESUMO I Um dos maiores problemas de saúde pública na população idosa são as quedas, agravando-se quando relacionadas à presença de osteoporose. Dentre os vários fatores de risco, destacam-se a diminuição do equilíbrio, controle postural e força muscular. O objetivo deste trabaIho foi comparar o equilíbrio, o controle postural e a força muscular em idosas osteoporóticas com e sem quedas referidas no último ano. Foram avaliadas 45 mulheres entre 65 e 85 anos, divididas em dois grupos com base no relato de quedas nos 12 meses anteriores à avaliação: grupo com quedas (GCQ; $n=21$ ) e grupo sem quedas (GSQ; $n=24$ ). $O$ equilíbrio foi avaliado por meio da escala de equilíbrio de Berg; o controle postural pelo teste clínico modificado de interação sensorial no equilíbrio (mCTSIB), realizado no equipamento Balance Master ${ }^{\circledR}$; e a força muscular dos flexores e extensores de joelho e dorsiflexores de tornozelo, com dinamômetro EMG System do Brasil ${ }^{\oplus}$. Foi considerado nível de significância $\alpha=0,05$. Houve diferença significativa no equilibrio $(p<0,01)$ e na velocidade de oscilação do Centro de Pressão (CP) durante o teste mCTSIB nas condições olhos fechados superfície estável $(p=0,05)$ e olhos abertos superfície instável $(p<0,01)$, com valores maiores para o GCQ. Os grupos foram semelhantes entre si em relação à força muscular $(p>0,05)$. Nossos resultados indicam que idosas osteoporóticas com histórico de quedas nos últimos 12 meses possuem pior equilíbrio e controle postural em relação às osteoporóticas sem quedas referidas.
\end{abstract}

Descritores I idoso; osteoporose; equilíbrio postural; acidentes por quedas; força muscular.

\begin{abstract}
I Falls are one of the biggest public health problems in the elderly population, worsening when related to the presence of osteoporosis. Among the various risk factors are highlighted decreased balance, muscle strength and postural control. This study aim is to compare the balance, postural control and muscle strength in osteoporotic elderly women with and without falls reported in the last year. We evaluated 45 women between 65 and 85 years, divided into two groups, based on reports of falls in the 12 months prior to assessment: group with falls $(n=21)$ and group without falls $(n=24)$. Balance was assessed using the Berg balance scale; postural control by the modified clinical test of sensory interaction on balance (mCTSIB); performed by equipment Balance Master ${ }^{\circledR}$, and muscle strength of knee flexors and extensors and ankle extensor by the dynamometer ${ }^{\circledR}$ EMG System of Brazil. We considered the level of significance $\alpha=0.05$. There were significant differences in balance $(p<0.01)$ and the speed of oscillation of the center of pressure (CP) during the test conditions mCTSIB eyes closed surface $(p=0.05)$ and unstable eyes open $(p<0.01)$, with higher values for the group with falls. The groups were similar to muscle strength ( $p>0.05$ ). Our results indicate that osteoporotic elderly women with a history of falls in the last 12 months have poor balance and postural control in elderly osteoporotic compared to those without falls.
\end{abstract}

Keywords I aged; osteoporosis; postural balance; accidental falls; muscle strength.

Estudo desenvolvido no Laboratório de Avaliação Fisioterapêutica Clínica e Eletromiográfica do Departamento de Fisioterapia, Fonoaudiologia e Terapia Ocupacional da Universidade de São Paulo (USP) - São Paulo (SP), Brasil.

'Fisioterapeuta graduada pela Faculdade de Medicina da USP - São Paulo (SP), Brasil.

²Doutorando em Fisiopatologia Experimental pela Faculdade de Medicina da USP - São Paulo (SP), Brasil.

${ }^{3}$ Professora Associada do Curso de Fisioterapia Faculdade de Medicina da USP - São Paulo (SP), Brasil. 


\section{INTRODUÇÃO}

Osteoporose é uma doença comum, caracterizada por diminuição da densidade mineral óssea (DMO) e deterioração na microarquitetura do tecido ósseo ${ }^{1}$. Estima-se que ela afete aproximadamente $55 \%$ da população acima dos 50 anos de idade nos Estados Unidos 2 , e 15\% da população brasileira ${ }^{3}$ com predileção pelo gênero feminino.

A diminuição da massa óssea, apesar de não ser um fator de risco para quedas, contribui significantemente para o risco de fratura. As mais comuns são as de quadril, punho e as vertebrais, consideradas típicas da osteoporose $e^{4}$. As consequências das fraturas podem ser sérias e estão associadas a altas taxas de mortalidade (33\% no primeiro ano) e aumento da morbidade ${ }^{5,6}$.

O controle postural deficitário é apontado como importante fator de risco para quedas em idosos, havendo indicativos de que diminui com o passar da idade ${ }^{7}$, aumentando a velocidade de oscilação do centro de pressão $(\mathrm{CP})$ durante a posição vertical ${ }^{8}$. Liu-Ambrose et al. ${ }^{9}$ e Burke et al. ${ }^{10}$ sugerem que idosos com osteoporose possuem, além da fragilidade mecânica resultante das alterações na microarquitetura óssea, risco de quedas e de fraturas aumentado por apresentarem diminuição mais pronunciada de força e equilíbrio em relação a idosos sem osteoporose.

Apesar das variáveis equilíbrio, força muscular e controle postural diferirem entre osteoporóticos e não osteoporóticos, essas características ainda não foram estudadas em grupos de idosos com osteoporose que caem e que não caem, havendo uma lacuna em relação ao tema. Portanto, este estudo teve como objetivo comparar o equilíbrio, controle postural e força muscular em idosas osteoporóticas com e sem quedas referidas no último ano.

\section{MATERIAIS E MÉTODOS}

\section{Delineamento: estudo transversal}

Participaram do estudo 45 mulheres com diagnóstico de osteoporose de colo de fêmur e/ou coluna lombar entre 65 e 85 anos, selecionadas a partir da lista fornecida pelo grupo do Laboratório de Metabolismo Ósseo do Serviço de Reumatologia da Faculdade de Medicina da Universidade de São Paulo (FMUSP).

Foram critérios de inclusão ter diagnóstico de osteoporose (DMO abaixo de 2,5, desvio-padrão em coluna lombar ou colo do fêmur total ${ }^{11}$, idade entre 65 e 85 anos e gênero feminino; e de exclusão, presença de doenças

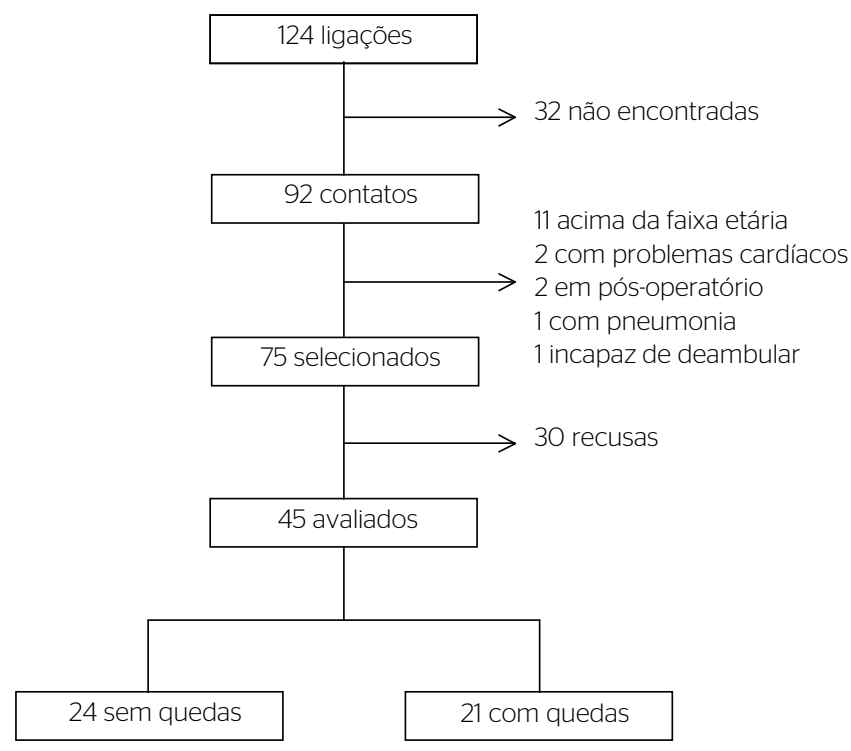

Figura 1. Fluxograma de captação dos participantes

neurológicas, deficiência visual grave, incapacidade física de participar dos testes, amputações ou uso de próteses de membros inferiores. A Figura 1 ilustra o fluxograma utilizado para entrar em contato com os participantes.

Após a aplicação dos critérios de inclusão e exclusão, as 45 participantes foram divididas de acordo com o relato de quedas nos 12 meses anteriores à avaliação: 24 foram alocadas no grupo sem quedas (GSQ) e 21 no grupo com quedas (GCQ).

O estudo foi aprovado pelo Comitê de Ética em Pesquisa do Hospital das Clínicas da FMUSP. Todos os participantes assinaram um Termo de Consentimento Livre e Esclarecido.

\section{Procedimento}

Foi utilizado questionário para obter dados sociodemográficos, características antropométricas e histórico de quedas. O equilíbrio funcional foi medido pela Escala de Equilíbrio de Berg, a força muscular isométrica dos membros inferiores por um dinamômetro (CRF200, EMG System do Brasil) e o controle postural pela plataforma de força Balance Master (NeuroCom), aplicando-se o teste clínico modificado de interação sensorial para equilíbrio (mCTSIB).

\section{Equilíbrio funcional}

Para a avaliação do equilíbrio funcional foi utilizada a Escala de Equilíbrio de Berg, validada para língua portuguesa por Miyamoto ${ }^{12}$. Ela atende várias propostas: descrição quantitativa da habilidade de equilíbrio funcional, acompanhamento do progresso dos pacientes 
e avaliação da efetividade das intervenções na prática clínica e em pesquisas. A escala avalia o equilíbrio funcional em 14 itens comuns à vida diária. Cada um deles possui uma escala ordinal de cinco alternativas, que variam de 0 a 4 pontos, com pontuação máxima de 56 .

\section{Controle postural}

Para a avaliação do controle postural, os sujeitos realizaram o mCTSIB em uma plataforma de força modelo Balance Master (NeuroCom). Ele mede o equilíbrio estático em quatro condições sensoriais: superfície estável e olhos abertos; superfície estável e olhos fechados; superfície instável e olhos abertos e superfície instável e olhos fechados. Os participantes foram testados em posição ortostática, com pés descalços e posicionados em uma das três posições recomendadas pelo fabricante, com os braços lateralmente ao corpo. A posição dos pés foi monitorada durante o teste. $\mathrm{O}$ experimento foi repetido três vezes, por dez segundos cada, e a média dos resultados foi considerada.

\section{Força muscular isométrica}

Foi medida a força muscular isométrica de dorsiflexão do tornozelo, e extensão e flexão do joelho. Solicitou-se aos voluntários realizar duas contrações (seis segundos cada) para cada teste, com 30 segundos de descanso. Um dinamômetro (CRF200, EMG System do Brasil) com sensibilidade de 0-100 kg foi fixado a uma parede e preso ao tornozelo ou ao dorso dos pés (dorsiflexão), utilizando-se uma faixa inextensível de tecido sintético. Para extensão e flexão dos joelhos, os participantes permaneceram sentados, com o tórax na posição vertical e os braços cruzados sobre o peito, sem tocar os pés no chão. Os joelhos foram mantidos a 90 graus. Para a dorsiflexão do tornozelo, os participantes permaneceram em decúbito dorsal, com o tornozelo em 110 graus de flexão plantar. Os sujeitos foram instruídos a usar a força máxima, assim que solicitado. A raiz quadrada média (RMS) foi considerada para cada uma das duas repetições.

\section{Análise estatística}

Para verificar a normalidade dos dados foi realizado o teste de Kolmogorov-Smirnov, e para comparar os grupos foi utilizado o teste $t$ de Student independente para variáveis paramétricas e o Mann-Whitney para as variáveis não paramétricas. Foi admitido nível de significância de 5\% $(\alpha=0,05)$.

\section{RESULTADOS}

A Tabela 1 descreve as características antropométricas das 45 participantes. Não houve diferença significativa entre os grupos ( $p>0,05)$, indicando que são semelhantes em relação à idade, peso, altura e índice de massa corpórea (IMC).

Já a Tabela 2 descreve os dados da escala de equilíbrio de Berg, força muscular isométrica e do mCTSIB. O GSQ apresentou valores mais altos em todas as variáveis, com diferença significativa na escala de Berg $(\mathrm{p}<0,05)$. A velocidade de deslocamento do $\mathrm{CP}$ foi maior nas condições no GCQ porém com diferença estatisticamente significativa para as condições 2 (olhos fechados e superfície estável) e 3 (olhos abertos e superfície instável) ( $p>0,05)$.

A Tabela 3 representa os dados do mCTSIB, que demonstram que o GCQ deslocou seu CP com mais velocidade durante as condições 2 (olhos fechados e superfície estável) e 3 (olhos abertos e superfície instável) do teste, indicando pior controle.

Tabela 1. Características antropométricas dos grupos e número de quedas

$\begin{array}{lccc}\text { Variáveis } & \begin{array}{c}\text { GSQ }(n=24) \\ \text { média } \pm D P\end{array} & \begin{array}{c}\text { GCQ }(n=21) \\ \text { média } \pm D P\end{array} & \text { Valor } p \\ \text { Idade (anos) } & 73,50 \pm 2,77 & 73,69 \pm 3,45 & 0,84 \\ \text { Massa corporal }(\mathrm{kg}) & 58,49 \pm 9,61 & 57,59 \pm 8,80 & 0,75 \\ \text { Altura }(\mathrm{m}) & 1,51 \pm 0,07 & 1,49 \pm 0,06 & 0,31 \\ \text { IMC }\left(\mathrm{kg} / \mathrm{m}^{2}\right) & 25,52 \pm 3,90 & 25,41 \pm 3,21 & 0,92 \\ \text { Quedas }(\mathrm{n}) & 0 & 1,00 \pm[2,12] & - \\ \text { (mediana } \pm[\mathrm{IC}]) & & \end{array}$

GSQ: grupo sem quedas; GCQ: grupo com quedas; DP: desvio-padrão; IMC: índice de massa corpórea; IC: intervalo de confiança

Tabela 2. Equilíbrio, força muscular isométrica e controle postural e comparação entre os grupos

\begin{tabular}{|c|c|c|c|}
\hline Variáveis & $\begin{array}{c}\text { GSQ }(n=24) \\
\text { média } \pm D P\end{array}$ & $\begin{array}{l}\mathrm{GCQ}(\mathrm{n}=21) \\
\text { média } \pm \mathrm{DP}\end{array}$ & Valor $\mathrm{p}$ \\
\hline Equilíbrio & $55,23 \pm 1,11$ & $52,43 \pm 3,89$ & $<0,01^{*}$ \\
\hline \multicolumn{4}{|l|}{$\mathrm{FMI}(\mathrm{kg} / \mathrm{f})$} \\
\hline Dorsiflexão de tornozelo & $12,82 \pm 3,82$ & $11,46 \pm 3,49$ & 0,25 \\
\hline Extensão de joelho & $19,44 \pm 6,01$ & $17,30 \pm 4,22$ & 0,21 \\
\hline Flexão de joelho & $8,58 \pm 3,76$ & $8,57 \pm 2,25$ & 0,99 \\
\hline \multicolumn{4}{|c|}{ Controle Postural (velocidade - \%s) } \\
\hline Condição $1^{\text {** }}$ & $0,24 \pm 0,09$ & $0,27 \pm 0,09$ & 0,30 \\
\hline Condição $2^{\star \star *}$ & $0,27 \pm 0,08$ & $0,39 \pm 0,23$ & $0,05^{*}$ \\
\hline Condiç̧ão $3^{\star * \star *}$ & $0,95 \pm 0,19$ & $1,39 \pm 0,58$ & $<0, \mathrm{O}^{*}$ \\
\hline Condição $4^{\star \star \star \star *}$ & $3,49 \pm 1,45$ & $3,89 \pm 1,79$ & 0,64 \\
\hline Composição & $1,23 \pm 0,39$ & $1,60 \pm 0,67$ & 0,10 \\
\hline
\end{tabular}

GSQ: grupo sem quedas; GCQ: grupo com quedas; DP: desvio-padrão,

*Estatisticamente significativo $(p<0,05)$; ${ }^{* *}$ Superfície firme com os olhos abertos; ${ }^{* * *}$ Superfície firme com os olhos fechados; ****Superfície instável com os olhos abertos; ****Superfície instável com os olhos fechados 
Tabela 3. Média e desvio-padrão da velocidade do Centro de Pressão de acordo com as condições do Modified Clinical Test of Sensory Interaction for Balance

\begin{tabular}{|c|c|c|c|}
\hline Variáveis ( $\%$ s) & $\begin{array}{l}\text { GSQ }(n=24) \\
\text { média } \pm D P\end{array}$ & $\begin{array}{l}\mathrm{GCQ}(\mathrm{n}=21) \\
\text { média } \pm \mathrm{DP}\end{array}$ & Valor $p$ \\
\hline Condição $1^{*}$ & $0,24 \pm 0,09$ & $0,27 \pm 0,09$ & 0,30 \\
\hline Condição $2^{* *}$ & $0,27 \pm 0,08$ & $0,39 \pm 0,23$ & $0,05^{\star \star \star}$ \\
\hline Condição $3^{\star \star \star \star ~}$ & $0,95 \pm 0,19$ & $1,39 \pm 0,58$ & $<0,01^{\star * \star}$ \\
\hline Condição $4^{\star \star * \star *}$ & $3,49 \pm 1,45$ & $3,89 \pm 1,79$ & 0,64 \\
\hline
\end{tabular}

GSQ: grupo sem quedas; GCQ: grupo com quedas; DP: desvio-padrão.

*Superficie firme com os olhos abertos; ** Superfície firme com os olhos fechados:

***Estatisticamente significativo $(p<0,05)$; *** Superfície instável com os olhos abertos;

****\$Superficie instável com os olhos fechados

\section{DISCUSSÃO}

O objetivo deste estudo foi comparar o equilíbrio, o controle postural e a força muscular em idosas osteoporóticas com e sem quedas referidas no último ano. Os dados sugerem que idosas com osteoporose com histórico de quedas apresentam pior equilíbrio e controle postural quando comparadas a idosas com osteoporose sem histórico de quedas. $\mathrm{Na}$ força muscular dos membros inferiores, não houve diferença entre os grupos.

$\mathrm{O}$ controle postural é uma medida do $\mathrm{CP}$ usada para determinar a estabilidade postural durante o equilíbrio estático ${ }^{13,14}$, e o teste clínico de interação sensorial para equilíbrio (CTSIB) avalia a oscilação postural projetada para medir a influência das informações sensoriais no equilíbrio $^{13,15}$, exigindo que o participante fique em pé em uma superfície estável e instável com os olhos abertos e fechados.

Nossos resultados apontam que o GCQ apresentou maior velocidade de oscilação em todas as condições do teste, sugerindo diminuição do equilíbrio, fato que pode ser atribuído à condição em que são realizados os testes. Por exemplo, a condição 1 (superfície estável e olhos abertos) acontece durante a maioria das atividades de vida diária e, por isso, é pouco sensível para captar diferenças; já a condição 4 (superfície instável e olhos fechados) é uma condição extrema na qual as aferências proprioceptivas e visuais são privadas e, portanto, uma situação que pode ter sido de grande dificuldade para ambos os grupos, igualando-os em seus resultados.

Segundo Choy et al. ${ }^{16}$, a velocidade de oscilação do $\mathrm{CP}$ aumenta com o passar da idade e parece ser um bom indicativo da capacidade de controle postural em idosos ${ }^{17}$. Wallmann ${ }^{18}$ comparou a oscilação entre idosos sem quedas com idosos caidores e, apesar das demais condições apresentarem aumento da oscilação nos caidores, somente a condição 3 teve diferença significante entre os grupos, fato que combina com os nossos resultados. Ao que parece, em contraste com os caidores recorrentes, os idosos sem quedas conseguem compensar de uma forma mais eficiente o conflito causado pela superfície instável no sistema somatossensorial.

Em relação ao equilíbrio funcional, nosso estudo aponta que o GCQ apresentou pontuação inferior ao GSQ na escala de Berg, o que indica pior equilíbrio. Ela tem sido utilizada para identificar idosos com grandes limitações e que possuem mais alto risco de cair que idosos ativos e com boa saúde, mas que também podem sofrer quedas ${ }^{19}$. Em nosso estudo, as idosas participantes eram independentes na locomoção e nas atividades de vida diária, o que pode explicar as pontuações encontradas, próximas dos valores máximos.

Obtivemos valores superiores a estudos como os de Shumway-Cook et al. ${ }^{20}$, Lajoie et al. ${ }^{21}$ e Lajoie et al. ${ }^{22}$. Essa diferença pode estar relacionada ao fato de que Shumway-Cook et al. ${ }^{20}$ classificaram caidores os idosos com quedas recorrentes relatadas nos últimos seis meses, e não nos últimos 12 como em nosso estudo. Nos estudos de Lajoie et al. ${ }^{23}$ e Lajoie et al. ${ }^{24}$ foram incluídos idosos tanto da comunidade quanto de instituições, o que pode ter contribuído para a menor pontuação.

Para Muir ${ }^{25}$, para aqueles que pontuam entre $50 \mathrm{e}$ 54 , o risco de cair é $16 \%$, diminuindo para $10 \%$ em pontuações acima de 55. Em nosso estudo, ambos os grupos obtiveram pontuações acima de 55 , portanto, diminuindo o risco de queda de 16 para $10 \%$. Um incremento de 1 a 2 pontos na escala pode ser significativo se considerarmos a gama de complicações ocasionadas pelas quedas na população idosa, principalmente naquela com osteoporose, em que as fraturas decorrentes de quedas são mais frequentes ${ }^{26}$.

Outro estudo ${ }^{27}$ identificou que pontuações de 41-56 indicam baixo risco de quedas; de 21-40, risco moderado; e abaixo de 20, risco alto. Nenhuma das idosas avaliadas em nosso estudo apresentou pontuação igual ou menor a 40 , sendo que o valor mais baixo foi de 42 pontos. Assim, todas se enquadram no risco baixo de quedas. Muir et al. ${ }^{26}$ concluíram que a Berg Balance Scale (BBS) tem melhor capacidade discriminatória para a identificação de idosos residentes na comunidade que apresentam recorrentes ou múltiplas quedas do que para identificar aqueles que caem uma vez.

Nossos dados demonstram que a força muscular foi semelhante entre os grupos, discordando dos resultados apresentados por Pijnappels et al. ${ }^{28}$, que encontraram diferença significativa para força muscular de extensão de joelho entre idosos caidores e não caidores. No entanto, Keskin et al. ${ }^{29}$ reforçam nossos achados, concluindo em 
seu estudo que a força dos extensores e flexores de joelho não tem efeito significativo na ocorrência ou risco de quedas em mulheres idosas, particularmente as que são funcionalmente independentes, característica semelhante às idosas de nosso estudo, uma vez que não necessitavam de auxílio em suas atividades de vida diária.

Segundo revisão sistemática de Moreland et al. ${ }^{30}$, a fraqueza muscular, principalmente de membros inferiores, pode ser considerada um fator de risco para quedas. A força diminui com a idade, e isso pode ser limitante em idosos na prevenção. A força máxima de extensão de joelho é considerada uma boa medida para identificar as pessoas com alto risco de cair ${ }^{31,32}$. Por meio de estudos cinéticos, já foi determinado que tanto o momento interno de extensão do quadril quanto o momento interno de dorsiflexão do tornozelo são significativamente menores em idosos caidores quando comparados com idosos sem história de quedas ${ }^{33,34}$.

\section{CONCLUSÃO}

Nossos resultados indicam que idosas osteoporóticas com histórico de quedas nos últimos 12 meses possuem pior equilíbrio e controle postural em relação a idosas osteoporóticas sem quedas referidas.

\section{REFERÊNCIAS}

1. Kanis JA, Melton LJ 3rd, Christiansen C, Johnston CC, Khaltaev N. The diagnosis of osteoporosis. J Bone Miner Res. 1994;9(8):1137-41.

2. Kuczynski M, Ostrowska B. Undersanding falls in osteoporosis: the viscoelastic modeling perspective. Gait Posture. 2006;23(1):51-8.

3. Costa-Paiva L, Horovitz AP, Santos AO, Fonsechi-Carvasan GA, Pinto-Neto AM. Prevalência de osteoporose em mulheres na pósmenopausa e associação com fatores clínicos e reprodutivos. Rev Bras Ginecol Obstet. 2003;25(7):507-12.

4. Kam D, Smulders E, Weerdesteyn V, Smits-Engelsman BC. Exercise interventions to reduce fall-related fractures and their risk factors in individuals with low bone density: a systematic review of randomized controlled trials. Osteoporos Int. 2009;20(12):2111-25.

5. Keene GS, Parker MJ, Pryor GA. Mortality and morbidity after hip fractures. BMJ. 1993;307:1248-50.

6. Roche JJ, Wenn RT, Sahota O, Moran CG. Effect of comorbidities and postoperative complications on mortality after hip fracture in elderly people: prospective observational cohort study. BMJ. 2005;331(7529):1374

7. Melzer I, Benjuya N, Kaplanski J. Postural stability in the elderly: a comparison between fallers and non-fallers. Age Ageing. 2004;33(6):602-7.
8. Hageman PA, Leibowitz JM, Blanke D. Age and gender effects on postural control measures. Arch Phys Med Rehabil. 1995;76(10):961-5

9. Liu-Ambrose T, Eng JJ, Khan KM, Carter ND, McKay HA. Older women with osteoporosis have increased postural sway and weaker quadriceps strength than counterparts with normal bone mass: overlooked determinants of fracture risk? J Gerontol A Biol Sci Med Sci. 2003:58(9):M862-6.

10. Burke TN, França FJ, Meneses SR, Cardoso VI, Pereira RM, Danilevicius CF, Marques AP. Postural control among elderly women with and without osteoporosis: is there a difference? Sao Paulo Med J. 2010;128(4):219-24.

11. World Health Organization. Assessment of fracture risk and application to screening for postmenopausal osteoporosis. Report of a WHO Study Group. World Health Organ Tech Rep Ser. 1994:843:1-129.

12. Miyamoto ST, Lombardi Junior I, Berg KO, Ramos LR, Natour J. Brazilian version of the Berg balance scale. Braz J Med Bio Res. 2004:37(9):1411-21

13. Nashner LM. Practical biomechanics and physiology of balance. In: Jacobson GP, Newman CW, Kartush JM, editors. Handbook of balance function testing. St. Louis: Mosby-Year Book; 1993. p. 261-79.

14. Rogers ME, Rogers NL, Takeshima N, Islam MM. Methods to assess and improve the physical parameters associated with fall risk in older adults. Prev Med. 2003;36(3):255-64.

15. Nashner LM, McCollum G. The organization of human postural movements: a formal basis and experimental synthesis. Behav Brain Sci. 1985:8:135-50.

16. Choy NL, Brauer S, Nitz J. Changes in postural stability in women aged 20 to 80 years. J Gerontol A Biol Sci Med Sci. 2003;58(6):525-30.

17. Melzer I, Benjuya N, Kaplanski J. Postural stability in the elderly: a comparison between fallers and non-fallers. Age Ageing. 2004:33(6):602-7.

18. Wallmann HW. Comparison of elderly nonfallers and fallers on performance measures of functional reach, sensory organization, and limits of stability. J Gerontol A Biol Sci Med Sci. 2001;56(9):M580-3.

19. Boulgarides LK, McGinty SM, Willett JA, Barners CW. Use of clinical and impaired-based tests to predict falls by community-dwelling older adults. Phys Ther. 2003;83(4):328-39.

20. Shumway-Cook A, Baldwin M, Polissar NL, Gruber W. Predicting the probability for falls in community-dwelling older adults. Phys Ther. 1997;77(8):812-9.

21. Lajoie Y, Girard A, Guay M. Comparison of the reaction time, the Berg Scale and the $A B C$ in non-fallers and fallers. Arch Gerontol Geriatr. 2002;35(3):215-25

22. Lajoie Y, Gallagher SP. Predicting falls within the elderly community: comparison of postural sway, reaction time, the Berg balance scale and the Activities-specific Balance Confidence (ABC) scale for comparing fallers and non-fallers. Arch Gerontol Geriatr. 2004:38(1):11-26.

23. Lajoie Y, Girard A, Guay M. Comparison of the reaction time, the Berg scale and the $A B C$ in non-fallers and fallers. Arch Gerontol Geriatr. 2002:35(3):215-25

24. Lajoie Y, Gallagher SP. Predicting falls within the elderly community: comparison of postural sway, reaction time, the Berg balance scale and the Activities-specific Balance Confidence (ABC) scale for comparing fallers and non-fallers. Arch Gerontol Geriatr. 2004;38(1):11-26. 
25. Muir SW, Berg K, Chesworth B, Speechley M. The use of the Berg balance scale for predicting multiple falls in communitydwelling elderly people: a prospective study. Phys Ther. 2008;88(4):449-59.

26. Hess JA, Woollacott M. Effect of high-intensity strength-training on functional measures of balance ability in balance-impaired older adults. J Manipulative Physiol Ther. 2005;28(8):582-90.

27. Whitney S, Wrisley D, Furman J. Concurrent validity of the Berg balance scale and the Dynamic gait index in people with vestibular dysfunction. Physiother Res Int. 2003;8(4):178-86.

28. Pijnappels M, van der Burg PJ, Reeves ND, van Dieën JH. Identification of elderly fallers by muscle strength measures. Eur J Appl Physiol. 2008;102(5):585-92.

29. Keskin D, Borman P, Ersöz M, Kurtaran A, Bodur H, Akyüz M. The risk factors related to falling in elderly females. Geriatr Nurs. 2008;29(1):58-63.
30. Moreland JD, Richardson JA, Goldsmith CH, Clase CM. Muscle weakness and falls in older adults: a systematic review and metaanalysis. JAGS. 2004;Suppl 2:1121-29.

31. Pijnappels M, Reeves ND, Maganaris CN, van Dieën JH. Tripping without falling; lower limb strength, a limitation for balance recovery and a target for training in the elderly. J Electromyogr Kinesiol. 2008:18(2):188-96.

32. Pijnappels M, van der Burg PJ, Reeves ND, van Dieën JH. Identification of high-risk fallers by force capacity measures in the elderly. J Biomech. 2006;39(Suppl 1):S87.

33. Kemoun G, Thoumie P, Boisson D, Guieu JD. Ankle dorsiflexion delay can predict falls in the elderly. J Rehabil Med. 2000;34(6):278-83.

34. Kirkwood RN, Araújo PA, Dias CS. Biomecânica da marcha em idosos caidores e não caidores: uma revisão da literatura. Rev Bras Ciênc Mov. 2006;14(4):103-10. 